\title{
Effect of Low Power Laser Irradiation on
}

\section{the Ability of Cell Growth and Myogenic Differentiation of Myoblasts Cultured In Vitro}

\author{
Cui-Ping Zhang, ${ }^{1}$ Shao-Dan Li, ${ }^{2}$ Xiao-Ying Wang, ${ }^{3}$ Peng Chen, ${ }^{4}$ Chang-Zhen Wang, \\ Xiao-Bing Fu, ${ }^{1}$ Hong-Xiang Kang, ${ }^{4}$ Ben-Jian Shen, ${ }^{4}$ and Jie Liang ${ }^{4}$ \\ ${ }^{1}$ Department of Wound Healing and Cell Biology, The First Affiliated Hospital, \\ General Hospital of Chinese People's Liberation Army, Beijing 100048, China \\ ${ }^{2}$ Institute of Traditional Chinese Medicine, General Hospital of Chinese People's Liberation Army, Beijing 100853, China \\ ${ }^{3}$ Department of Endocrinology, Navy General Hospital of Chinese People's Liberation Army, Beijing 100048, China \\ ${ }^{4}$ Department of Electromagnetic and Laser Biology, Institute of Radiation Medicine, \\ Academy of Military Medical Sciences, Beijing 100850, China
}

Correspondence should be addressed to Peng Chen; chenbj2002@sina.com

Received 30 April 2014; Revised 5 June 2014; Accepted 5 June 2014; Published 8 July 2014

Academic Editor: Gerhard Litscher

Copyright (c) 2014 Cui-Ping Zhang et al. This is an open access article distributed under the Creative Commons Attribution License, which permits unrestricted use, distribution, and reproduction in any medium, provided the original work is properly cited.

As a therapeutic modality, low power laser irradiation (LPLI) has been used clinically in the treatment of skeletal muscle injuries and other myopathic conditions, but the cellular and molecular mechanisms attributed to this therapy were still unclear. Myoblasts are a type of myogenic stem cells quiescence in mature skeletal muscle fibers and are considered as the source cells during the regenerating process. The purpose of this paper was to investigate the effects of LPLI on the proliferation and myogenic differentiation of the cultured myoblasts and to find out the major candidates responsible for LPLI-induced muscle regeneration in vivo. In this study, primary rat myoblasts were exposed to helium-neon (He-Ne) laser. Cell proliferation, differentiation, and the cellular responses to LPLI were monitored by using morphological observation and molecular biological methods. It was found that LPLI at a certain fluence could increase the cell growth potential for myoblasts and further induce more cells entering into $\mathrm{S}$ phase of the mitotic cycle as indicated by high levels of bromodeoxyuridine (BrdU) incorporation, while at the same time inhibiting their in vitro differentiation and decreasing the expression of myogenic regulatory genes to a certain extent. Taken together, these results provide experimental evidence for the clinical applications of LPLI in regenerating skeletal muscle.

\section{Introduction}

The primary functions of skeletal musculature are locomotor activity, postural behavior, and breathing. However, skeletal muscle is susceptible to injury after direct trauma or resulting from indirect causes, such as neurological dysfunction or innate genetic defects [1]. If left unrepaired, these injuries may lead to loss of muscle mass, locomotive deficiency, and in the worse cases lethality. Nowadays, many therapeutic approaches, such as cryotherapy, thermotherapy, electrotherapy, ultrasound, and medication, have been used to improve muscle healing process [2], but few of them are effective.
Low power laser is usually a laser with milliwatt-grade output power which can produce special biological stimulation for disease treatment rather than resulting in pathologic damage of tissues [3, 4]. LPLI has achieved positive effects in clinical treatment of wound healing, chronic pain relief, periodontal disease, and fracture rehabilitation [5-8]. Recent in vivo studies have showed that, following LPLI, the injured skeletal muscle recovered much faster and survived longer than unirradiated tissues, indicating that LPLI can be utilized for muscle physiological regeneration and functional reconstitution [9]; however, the cellular and molecular mechanisms of the laser-induced photobiomodulation attributed to this therapy are still unclear. 
It is well known that skeletal muscle repair is a complex and dynamic process involving the activation of various cellular responses [1]. Within these cellular events, myogenic progenitor cells, also termed myoblasts, play a crucial role in this healing process [10]. In the early stages of body development, myoblasts can continue to proliferate and integrate into multinucleated myotubes, thus leading to the formation of young skeletal muscle fibers and facilitating postnatal myogenesis in vivo. Yet, in adult skeletal muscle, the number of myoblasts was few and most of them were in quiescence, which made tissue regeneration and function repair very difficult after skeletal muscle injury $[10,11]$.

Recent studies have shown that, upon injury and with exogenous stimulus such as growth factors, myoblasts have the remarkable ability to initiate mitotic cycle and then begin to proliferate to form new multinucleated myotubes thus preventing the loss of muscle mass after muscle trauma [11]. Previous results have demonstrated that LPLI could markedly promote the recovery of injured skeletal muscle $[9,12]$. Obviously, the pathological basis for the laserenhanced repair process must be local cell proliferation in irradiation region. However, there is uncertainty in the present regarding whether myoblasts were also involved in this process. Therefore, in the present study, we used myoblasts cultured in vitro to investigate the effect of LPLI on myoblasts proliferation as well as their myogenic differentiation and to further reveal the possible molecular mechanisms for LPLI-promoted skeletal muscle regeneration after myopathic disorders. This study may possibly provide experimental evidence for the therapeutic use of LPLI in clinical practice.

\section{Materials and Methods}

2.1. Morphological Observations and Identification of Myoblasts. Myoblasts were isolated from the hind-limb muscles of neonatal Wistar rats and enriched by the preplating method according to literature [13]. The cells were cultured in poly-L-lysine coated Petri dishes containing Ham's F-10 nutrient medium supplemented with $20 \%$ fetal bovine serum (FBS), antibiotics $(100 \mathrm{U} / \mathrm{mL}$ penicillin $\mathrm{G}$, and $100 \mu \mathrm{g} / \mathrm{mL}$ streptomycin), and L-glutamine ( $4 \mathrm{mmol} / \mathrm{L})$. Cell morphology and myogenic differentiation were observed on the cultures using an inverted light microscope. For cell specificity analysis, the cells were fixed with $4 \%$ paraformaldehyde $\left(4^{\circ} \mathrm{C}\right.$, $2 \mathrm{~h}$ ), permeabilized with $1 \%$ Triton X-100 (room temperature, $10 \mathrm{~min}$ ), and blocked with $3 \%$ goat serum for $30 \mathrm{~min}$. The cells were then reacted with FITC- (fluorescein isothiocyanate-) labeled rabbit polyclonal antibody against desmin (Abcam, RS-91026R-FITC), which was diluted in 0.01 M PBS to the concentration of $1: 50$ and counterstained with Hoechst 33258 for $15 \mathrm{~min}$ to highlight nuclei. Finally, the immunoreactivity was monitored using a Carl Zeiss scanning confocal microscope (LSCM). Images were scanned and $0.32 \mathrm{~mm}$ serial optical sections were acquired. Composite images of the sample were assessed for estimation of the percentage of myogenic cells.
2.2. Laser Irradiation Protocol. After several passages, the expanded primary cultures, which consisted of $95-100 \%$ desmin-positive cells, were subjected to laser irradiation. A He-Ne laser (Institute of Semiconductors, Chinese Academy of Sciences, China) with a wavelength of $632.8 \mathrm{~nm}$ was used in this study. When the cells covering the culture plate reached a semiconfluent monolayer, irradiation was performed. In our experiments, laser irradiation was delivered to the culture plate with a spot size of $3.3 \mathrm{~cm}$ diameter, covering the entire surface of the culture dish uniformly. Power output was kept constant at $55 \mathrm{~mW}$ in the continuous wave mode. The power density of irradiation on the cells was measured to be $6 \mathrm{~mW} / \mathrm{cm}^{2}$. The irradiation time was set to be $1 \mathrm{~min}, 3 \mathrm{~min}$, and $10 \mathrm{~min}$ comprising $0.36,1.08$, or $3.6 \mathrm{~J} / \mathrm{cm}^{2}$, respectively. Nonirradiated control cells were kept under the same conditions as their treated counterparts except for the irradiation; they were kept in the laminar flow cabinet beside the irradiated cultures.

2.3. Cell Count. Myoblasts were seeded in $35 \mathrm{~mm}$ plates at a density of $4 \times 10^{4}$ cells $/ \mathrm{mL}$ and then irradiated by laser once a day for three days. After that, cells were trypsinized and made into single cell suspension. Double-blind counting under microscope was performed with a hemocytometer. The presented data were from at least three independent experiments.

2.4. Myogenic Differentiation Analysis. The cells were firstly plated in $35 \mathrm{~mm}$ Petri dish containing growth medium. When grown to confluence, or nearly confluent, the cell cultures were switched from growth medium to differentiation medium \{DMEM (Dulbecco's modified eagle medium) supplemented with $2 \%$ HS (horse serum) and antibiotics (100 U/mL penicillin $\mathrm{G}$ and $100 \mu \mathrm{g} / \mathrm{mL}$ streptomycin)\}. Laser irradiation was then conducted as described above (once every day for 3 days). After laser irradiation, hematoxylin and eosin (HE) staining was performed at room temperature. Cells induced by differentiation medium were fixed with $95 \%$ ethanol for $20 \mathrm{~min}$ and stained with hematoxylin for $3 \mathrm{~min}$ and with eosin for $1 \mathrm{~min}$, respectively. Myogenic differentiation was observed by light microscopy. Myogenic differentiation was represented by myotube formation rate (MFR), which was defined as the ratio of the number of nuclei within the integrated multinucleated myotubes to the total nuclei.

2.5. Cell Pretreatment and Grouping. To analyze the effect of laser irradiation on myoblasts individually and eliminate the possible impact of unknown factors in serum on the experiment results, cell pretreatment with serum starvation was introduced in the following experiment. Briefly, myoblasts were initially plated in the growth medium supplemented with $20 \%$ FBS overnight and then the cell cultures were replaced by serum-free medium for an additional $36 \mathrm{~h}$. Serum-free medium can only maintain in vitro survival of myoblasts rather than promoting their proliferation. The cells rendered quiescence by serum deprivation were then either refed with $10 \%$ FBS (hereinafter referred to as "10\% FBS 
group") or irradiated as described above "laser group." The cells neither refed nor irradiated were hereinafter referred to as "control group." After serum starvation, myoblasts in laser and control group were kept in the serum-free medium continuously. Meanwhile, the cells without any treatment (neither starved nor irradiated) were used as a positive control (20\% FBS group) in this study.

2.6. Flow Cytometric Analysis of BrdU Incorporation. Immediately after irradiation/refeeding, $20 \mu \mathrm{L}$ bromodeoxyuridine (BrdU) was added into the culture medium of all experimental groups for $20 \mathrm{~h}$ incubation. After fixation and centrifugation, the cells were resuspended in phosphatebuffered saline (PBS) and then incubated with a mouse monoclonal anti-BrdU (Boster, BM0201) overnight at $4^{\circ} \mathrm{C}$, followed by incubation with FITC-conjugated secondary antibody (Boster, BA1101) for $30 \mathrm{~min}$. After washing twice with PBS, flow cytometric assay (FACSCalibur, BD) was performed and the levels of BrdU incorporation (as indicated by mean fluorescence intensity) were calculated by the preloaded CELLQuest software for each sample.

2.7. Immunohistochemical Staining. For determination of myosin expression, myoblasts were fixed with $4 \%$ paraformaldehyde and then blocked with $3 \% \mathrm{H}_{2} \mathrm{O}_{2}$ to exclude nonspecific reaction. After thorough washing with PBS, cells were incubated with rabbit antibody against myosin (Santa Cruz, sc-66979, $1: 1000$ in PBS) at $4^{\circ} \mathrm{C}$ for $20 \mathrm{~h}$, followed by sequential incubation with biotinylated goat secondary antibody (Abbkine, A21220, 1:200 in PBS) and streptavidinhorseradish peroxidase (HRP) complex. The peroxidase reaction was developed using 3-amino-9-ethylcarbazol (AEC) as a chromogen and the immunostained sections were screened under a light microscope.

2.8. Western Blot Immunoassay. Expression of myogenic regulatory factors (MRFs) in myoblasts after LPLI was detected by western blotting. Briefly, cells were harvested after irradiation/refeeding at the desired time points and the total protein was extracted with lyses buffer as described previously [14]. Equal amounts of extracted proteins (total protein: $100 \mu \mathrm{g}$ ) were separated by SDS-PAGE and transferred onto nitrocellulose membranes using a semidry transfer (Bio-Rad). Membranes were blocked overnight at $4^{\circ} \mathrm{C}$ in blocking buffer and then incubated sequentially with primary antibodies to MyoD (Santa Cruz, sc-760, rabbit, 1:1000) and myogenin (Santa Cruz, sc-12732, mouse, 1:1000) for $3 \mathrm{~h}$ and HRP conjugated secondary antibodies (Santa Cruz, $1: 2000)$ for $2 \mathrm{~h}$. Protein expression was visualized using diaminobenzidine as chromogen. The relative gray values of corresponding bands and $\beta$-actin were then semiquantified and compared by Scion Image analysis software.

2.9. Statistical Analysis. All the experiments were repeated at least three times, unless otherwise indicated. Experimental data were presented as mean $\pm \mathrm{SD}$ (standard deviation) and statistical analysis was performed by $t$-test. A statistical probability of $P<0.05$ was considered significant.

\section{Results}

3.1. Morphological Features of Myoblasts Growth and Differentiation. Observed by light microscopy, the primary cells cultured in growth medium gradually became flat and spindle in shape after adherence. They proliferated, migrated, and were lined up regularly in one direction. When grown to nearly confluent and switched to serum-deficient differentiation medium, myoblasts could gradually form bifurcate and multinucleated myotubes with the presence of myonuclei above or beneath the basement membrane, some of which contracted spontaneously. Immunocytochemical results showed that the cell cultures were highly enriched for myogenic cells, approximately $95 \%$, as determined by desmin staining, a specific marker for myogenic cells (Figure 1). These primary myoblasts cultured in vitro could be expanded beyond 30 cell doublings without losing myogenic differentiation capacity, thus being available for the following experiments.

3.2. Effect of LPLI on Myoblasts Growth Capacity and Differentiation Ability. The cells were irradiated once every day as described above when reaching semiconfluency in $20 \%$ FBS growth medium. Three days after irradiation, cell counting results showed that irradiation for $3 \mathrm{~min}$ increased cell yield compared with control group, whereas the number of myoblasts receiving $10 \mathrm{~min}$ irradiation decreased notably when compared to nonirradiated cells (Figure 2).

In this study, we also investigated the impacts of LPLI with different irradiation times on myogenic differentiation ability. As shown in Figure 3, switching to differentiation culture medium with high cell seeding densities induced a significant differentiation of myoblasts to multinucleated myotubes. However, myotube differentiation was inhibited notably by $3 \mathrm{~min}$ laser irradiation, despite no significant difference between the other two laser-treated groups irradiated for either $1 \mathrm{~min}$ or $10 \mathrm{~min}$ and the nonirradiated control group.

3.3. LPLI Induced Myoblasts Entering into S Phase of the Mitotic Cycle. In this part, we assessed the effect of LPLI on the DNA-synthesis activity and cell proliferation cycle of myoblasts by detection of BrdU incorporation. BrdU is an analogue of thymine oligodeoxynucleotide; when cocultured with the cells in DNA-synthetic phase (S phase), BrdU can be inserted into the DNA chain which is replicating both in vivo and in vitro and thus may be used with the S-phase fraction as a measure of cell proliferation. As shown in Figures 4 and 5, in untreated control group, the majority $(>90 \%)$ of myoblasts did not incorporate any BrdU, while, in laser-irradiated and serum-refed group, approximately $50 \%$ and $63 \%$ of these cells were positive with much higher levels (mean fluorescence intensity) of BrdU incorporation, despite being still lower than the value of over $80 \%$ in the $20 \%$ FBS group.

\subsection{LPLI Inhibited Myoblasts Differentiation In Vitro}

3.4.1. Decreased Expression of Myosin in Response to LPLI. Beyond morphological changes and myotube formation, 


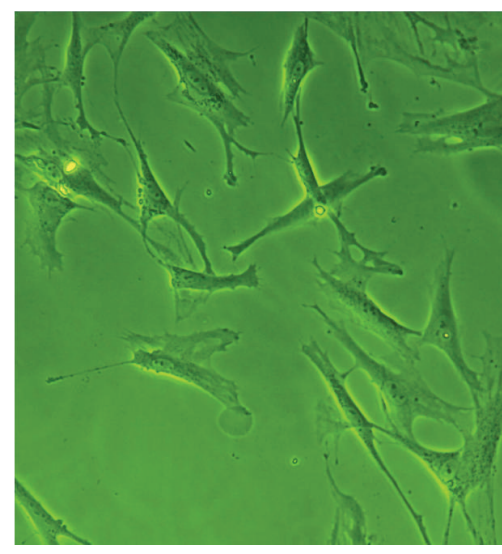

(a)

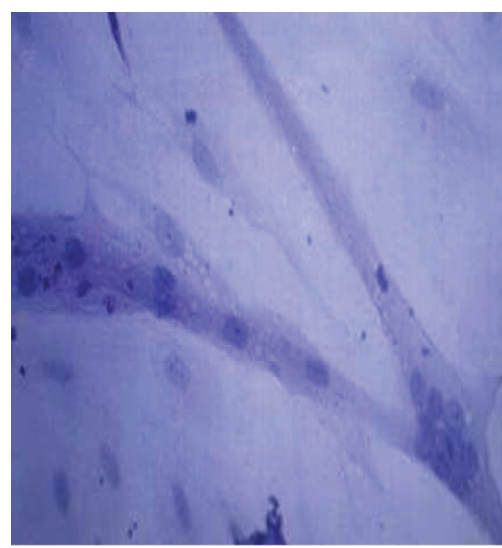

(d)

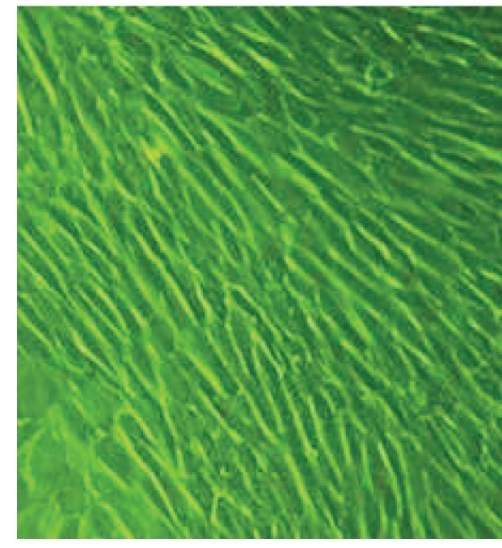

(b)

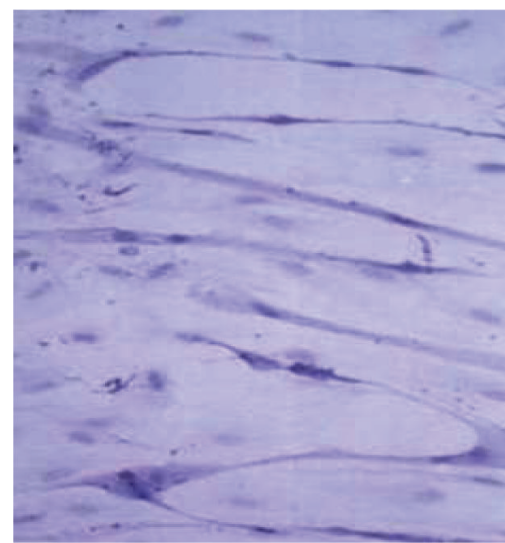

(e)

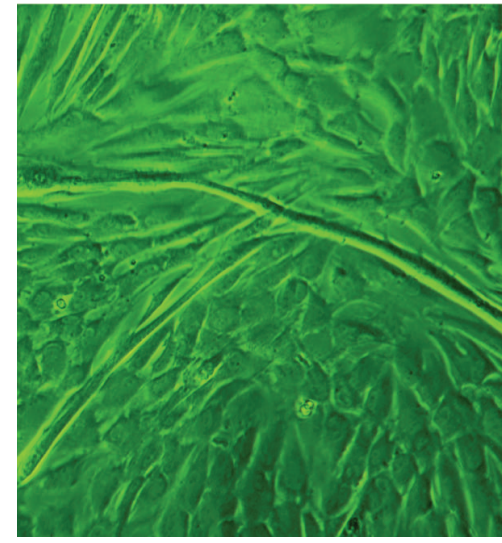

(c)

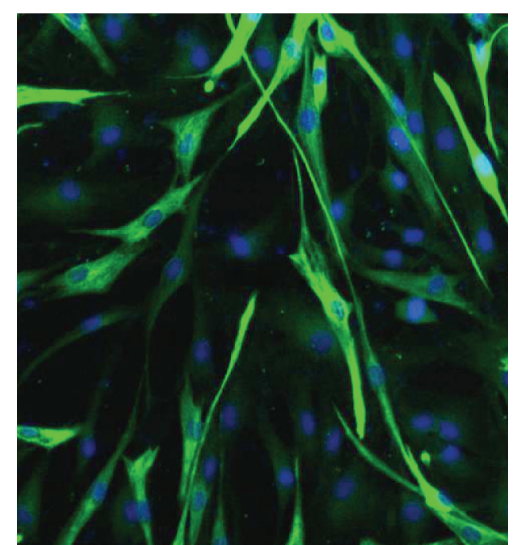

(f)

FIGURE 1: Morphological features and immunocytochemical identification of the primary myoblasts under inverted microscope and LSCM, respectively, $(\mathrm{a}, \mathrm{b})$ characteristic morphology of primary cultured myoblasts; (c) differentiated myoblasts to form linear and bifurcate myotubes; ( $\mathrm{d}, \mathrm{e}$ ) multinucleated myotubes visualized by HE staining; (f) myoblasts with desmin-positive expression presented double green and blue fluorescence under LSCM.

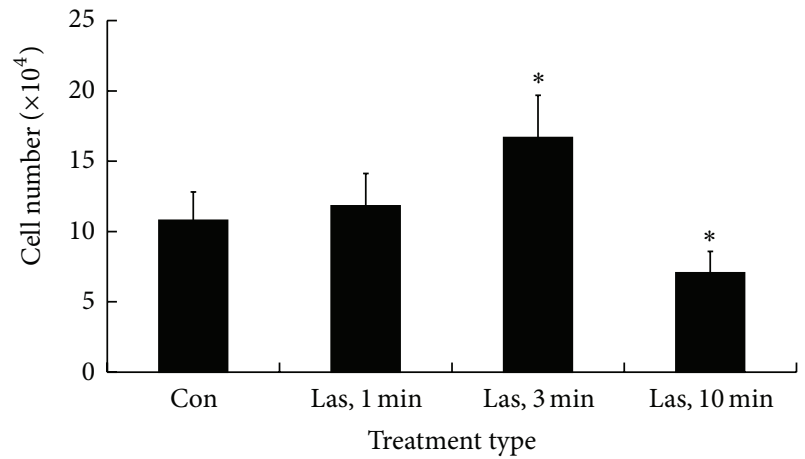

FIGURE 2: Effects of LPLI with different irradiation times on myoblasts growth capacity by cell counting. The data were from three independent experiments. Each bar represents mean \pm SD. ${ }^{*} P<0.05$ as compared with control group. Con: control; Las: laser irradiation.

synthesis, and expression of myosin, a skeletal musclespecific functional protein is also an important marker for myogenic differentiation of myoblasts [11]. Immunohistochemical staining results showed that the myosin expression in irradiation group was obviously lower than that in control

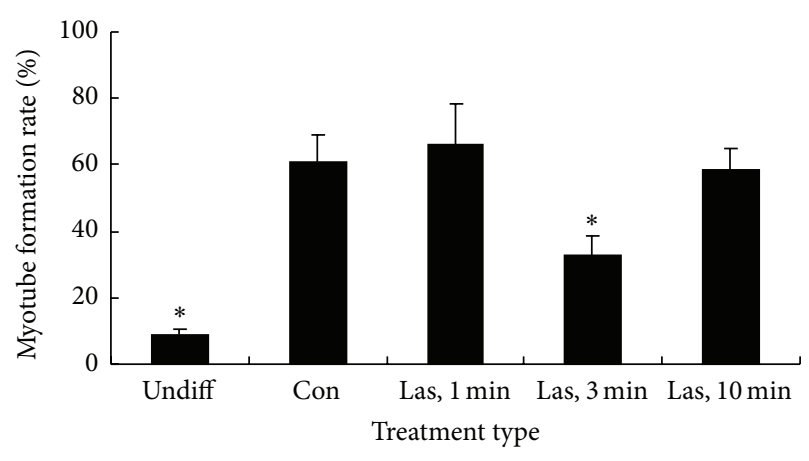

FIGURE 3: Effects of LPLI with different irradiation times on myoblasts myogenic differentiation ability as indicated by myotube formation rate (MFR). The data were from three independent experiments. ${ }^{*} P<0.05$ as compared with control group. Undiff: undifferentiated cells cultured in growth medium; Con: control; Las: laser irradiation.

group $48 \mathrm{~h}$ after irradiation/refeeding, but higher than that in $20 \%$ FBS group and 10\% FBS group, suggesting that LPLI could also inhibit in vitro myogenic differentiation 


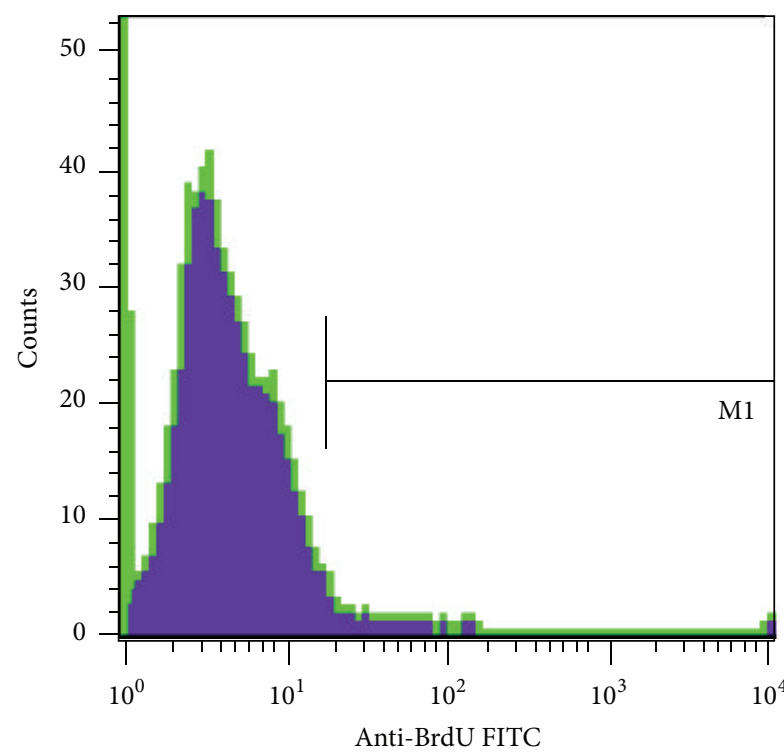

(a)

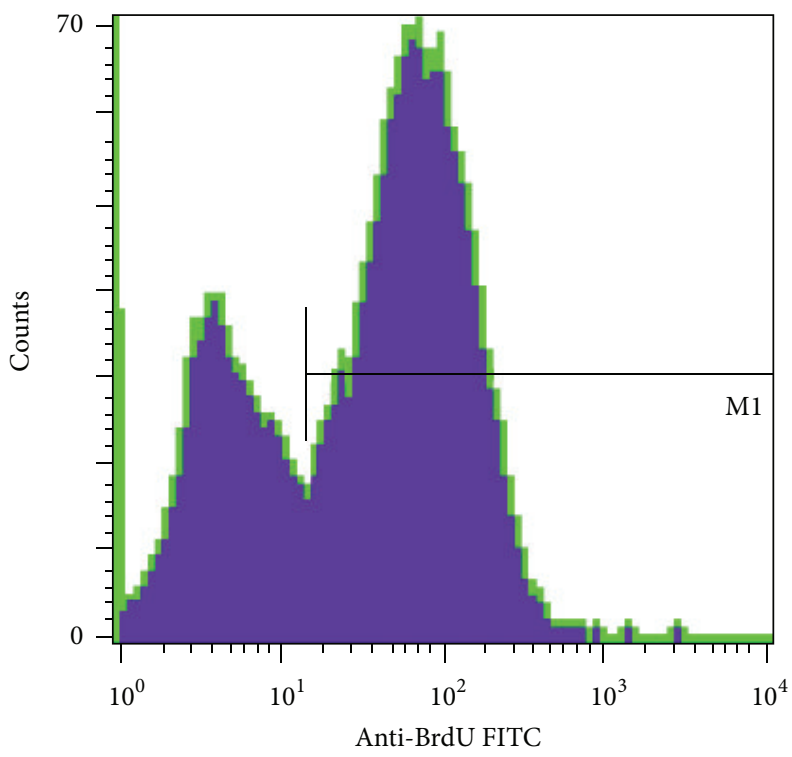

(c)

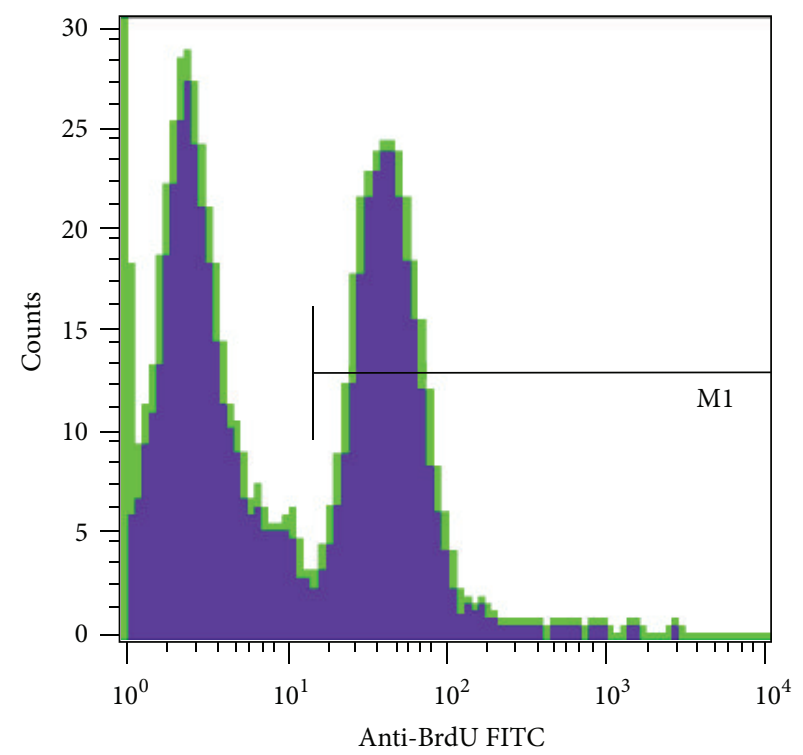

(b)

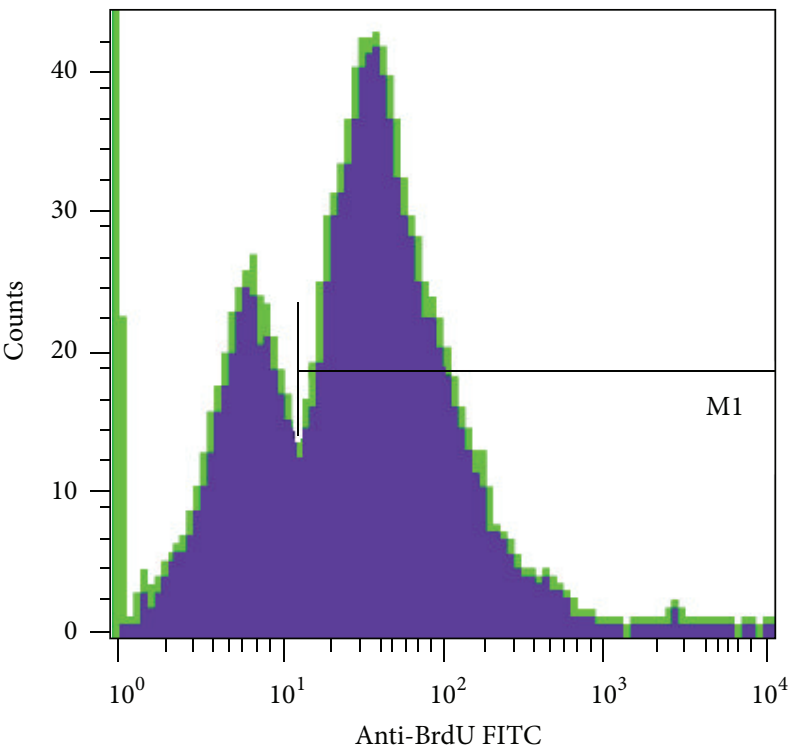

(d)

FIGURE 4: Effects of LPLI on the number of myoblasts in S phase of the cell cycle by flow cytometric analysis of BrdU incorporation after pretreatment with serum starvation. Representative histograms of flow cytometry analysis for BrdU-labeled cells from control (a), laser irradiation for $3 \mathrm{~min}$ (b), 10\% FBS (c), and 20\% FBS (d) group, respectively.

of myoblasts to a certain extent, while at the same time promoting them to enter into mitotic cycle (Figure 6).

\subsubsection{Effect of LPLI on Induction of Myogenic Regulatory} Factors (MRFs) of Myoblasts. It has been known that skeletal muscle differentiation is governed by tight regulation of both activity and expression levels of a number of transcription factors, particularly the MRFs family members (MyoD, Myf5, Myogenin, and MRF4) [12]. Among these, MyoD and myogenin were closely related to regulation in initiation of myogenesis and myogenic differentiation. Therefore, detection of these two gene expressions can reflect myoblasts differentiation ability. As shown in Figure 7, compared with the control group, MyoD and myogenin expressions were significantly reduced $24 \mathrm{~h}$ after irradiation/refeeding in laser group and $10 \%$ FBS group, their relative values being the same as the $20 \%$ FBS group.

\section{Discussion}

As a therapeutic modality, LPLI has been used clinically to improve the healing of a variety of myopathic conditions $[9,15]$. It has been demonstrated that the major factors responding to laser irradiation in regenerating muscles may 


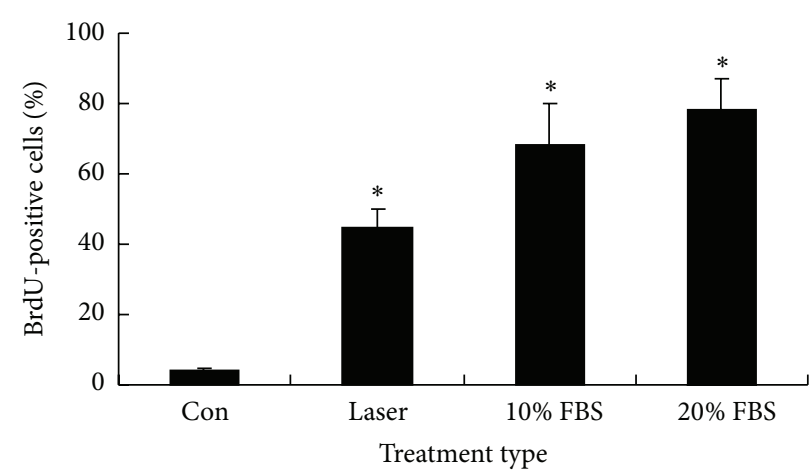

FIGURE 5: Cartogram of flow cytometric analysis regarding the percentage of BrdU-positive cells as represented by mean fluorescence intensity from the above groups, indicating that the number of cells in $\mathrm{S}$ phase was significantly increased after irradiation/refeeding treatment. $*$ Versus control, $P<0.05$. Con stands for control.

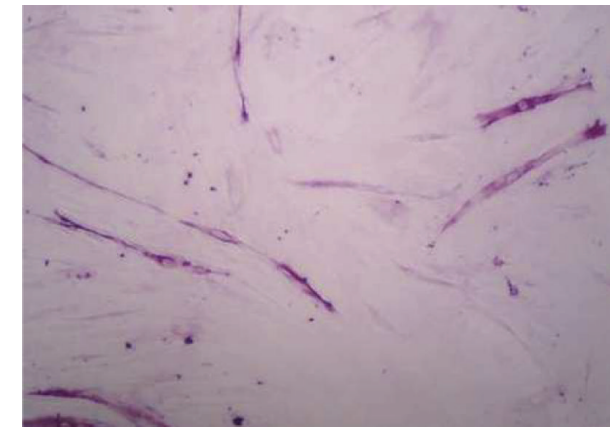

(a)

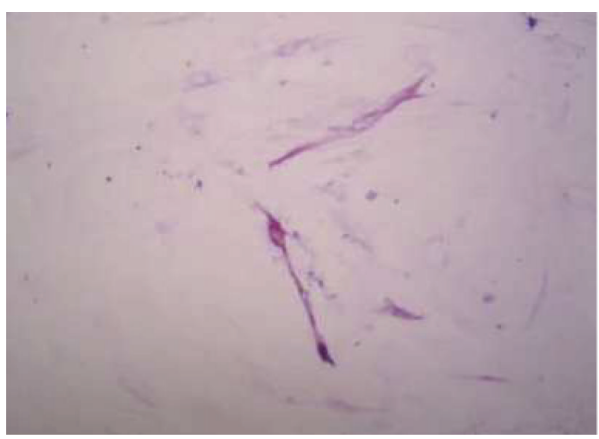

(c)

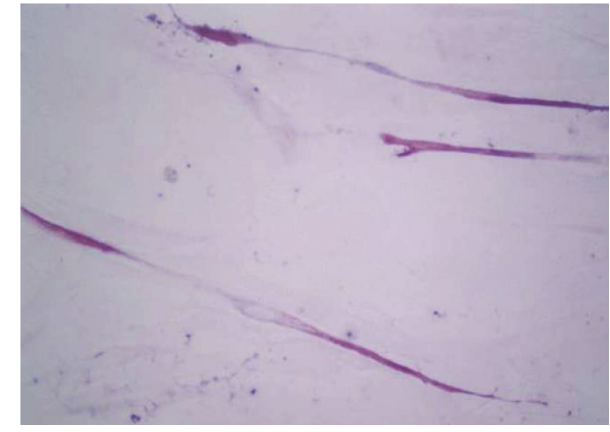

(b)

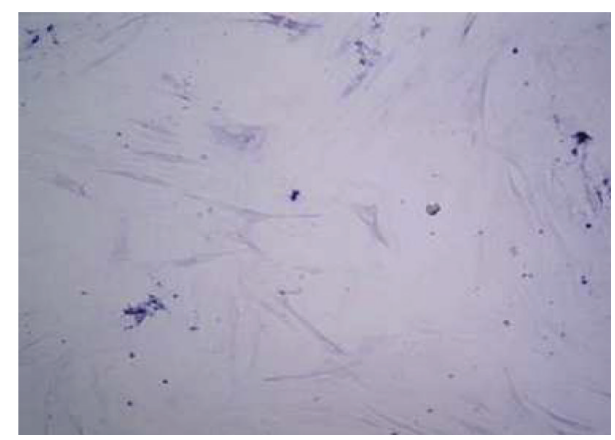

(d)

Figure 6: Expression of myosin in myoblasts in response to LPLI. The cells were treated and grouped as described above and expressions of myosin were determined $48 \mathrm{~h}$ after irradiation by immunohistochemistry. (a), (b), (c), and (d) were from control, laser irradiation for 3 min, $10 \%$ FBS, and $20 \%$ FBS group, respectively.

also be myoblasts [16]. In this study, the primary cultured myoblasts derived from the hind legs of neonatal rats were used for exploration of laser-induced biostimulatory effect. Firstly, we observed the impact of a He-Ne laser with different irradiation times on the growth and differentiation potential of the cultured cells, so as to identify the optimal treatment dosage for LPLI exerting maximal photobiostimulation on myoblasts. Between 1 and 5 days after laser irradiation, cultures were examined daily with phase-contrast optics for either cell counting or the presence of multinucleated cells. As shown above, laser irradiation for $3 \mathrm{~min}$ increased the cell number, whereas, for $1 \mathrm{~min}$ or $10 \mathrm{~min}$ irradiation, laser may have produced different and even opposite effects on myoblasts growth. In addition, myoblasts in control group were induced to differentiate into multinucleated myotubes by switching to differentiation culture media; however, the myotube formation could be inhibited to a certain extent by $3 \mathrm{~min}$ laser irradiation. These results above suggested that LPLI at certain fluence could increase in vitro growth capacity of myoblasts, while at the same time decreasing their myogenic differentiation.

Studies showed that the LPLI-induced biomodulatory effect was closely related to local microenvironment of the irradiated cells; that is, the initial physiological state of the cultured myoblasts is critical for the cellular response to LPLI [17-19]. Many negative reports are confusing primarily 


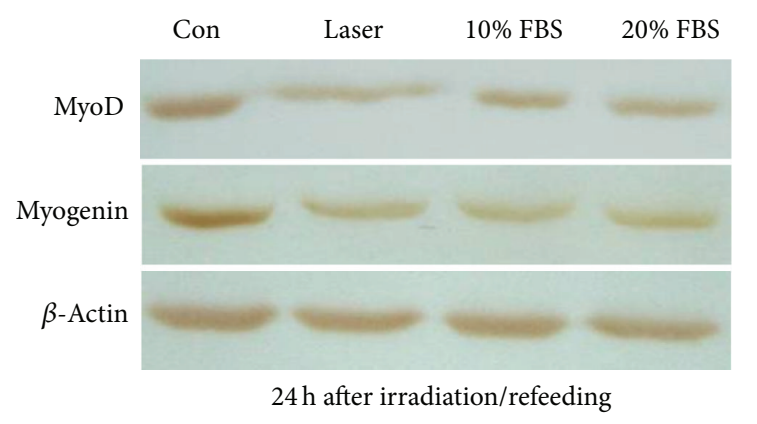

(a)

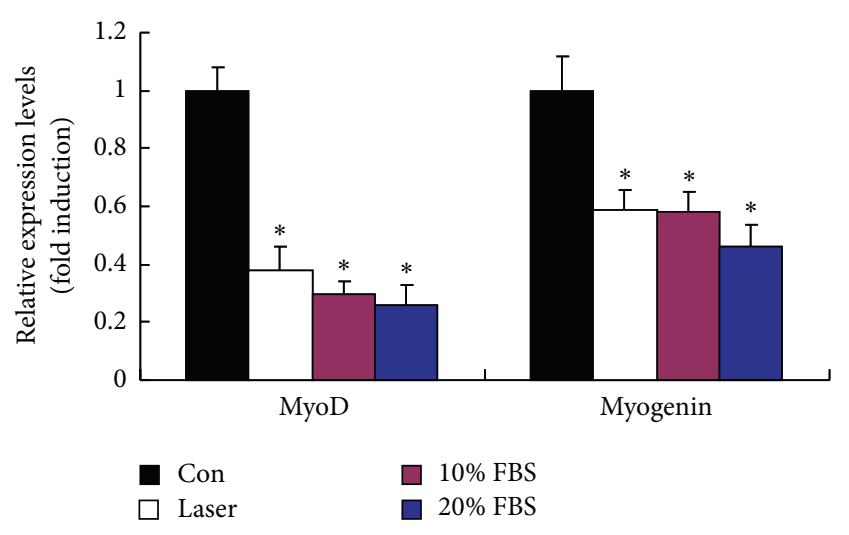

(b)

FIGURE 7: Effect of LPLI on MRFs expressions. Myoblasts were treated as above and harvested at the indicated time point followed by Western blotting. Blots were probed with antibodies against MyoD and myogenin, respectively (a). Relative expressions of MRFs were quantified by densitometric analysis and normalized to that of $\beta$-actin. The data were presented relative to control group arbitrarily normalized to 1 (b). ${ }^{*} P<0.05$ as compared with control group. Con stands for control.

because they do not include the dose response or pay attention to the initial state of the target cells. Thus in the following experiment, the protocol of pretreatment with serum starvation was introduced prior to laser irradiation. Serum starving is a common technique for growth arresting in cultured cells and decreasing their overall redox potential in order to reproduce the quiescent state of stem cell in vivo [20]. Therefore, after serum starvation, myoblasts were mostly synchronized in G0/G1 phase of the cell cycle, and so the cellular response to LPLI could be distinguished accurately.

The results of flow cytometric assay showed that, after pretreatment with serum starvation, laser irradiation for 3 min or serum refeeding could significantly increase BrdU incorporation levels which mark cells in $S$ phase of the mitotic cycle [21], suggesting that more myoblasts reentered into growth phase from quiescence and in some ways LPLI seems to be analogous to the essential components of growth medium required for myoblasts survival and growth in vitro. Meanwhile, we also found that laser irradiation of the same fluence could also reduce the myosin expression and MRFs activity, indicating that, in addition to enhancing myoblasts proliferation capacity, LPLI could also inhibit their differentiation into myotube in vitro, thereby giving rise to more myogenic descendants which could eventually facilitate muscle regeneration process in vivo.

Furthermore, in our unpublished article (date not shown), we found that laser irradiation for $3 \mathrm{~min}$ could also promote the expressions of cyclin D and cyclin A, two key cell-cycle regulatory proteins in myoblasts. In our experiment, a seesaw-like change of the expressions for both cyclins and MRFs was consistent with the biological properties of myoblasts [22]. The main reason for this phenomenon is that cyclin-CDKs (cyclin-dependent kinases) complexes could inhibit, whereas MRFs activate the skeletal muscle-specific gene transcription during myoblasts proliferation and differentiation [12, 23]. Studies have shown that MRFs, together with cell-cycle regulatory proteins, could coordinate cell cycle progression of myoblasts in vitro and these two proteins regulated proliferation and differentiation of myoblasts by oppositely affecting their cellular downstream targets such as retinoblastoma protein [22]. However, so far, the exact molecular mechanisms regarding the LPLI-induced biomodulatory effect on myoblasts and the possible influencing factors associated with this process have not been completely established and there is need for further exploration in future studies [24].

\section{Conclusion}

In summary, this experiment demonstrated that LPLI at a certain fluence could induce myogenic progenitor cells (myoblasts) entering into DNA-synthetic phase (S phase) of the mitotic cycle and to some extent inhibit ex vivo differentiation into myotube simultaneously and thus enhance their potential for cell growth and the ability to self-renew. These results indicated that LPLI may activate these myogenic stem cells from initial physiological "resting state" in vivo, thereby giving rise to more new myogenic descendants and hence facilitating muscle regeneration in clinical treatment.

\section{Conflict of Interests}

The authors declare that there is no conflict of interests regarding the publication of this paper.

\section{Authors' Contribution}

Cui-Ping Zhang, Shao-Dan Li, and Xiao-Ying Wang contributed equally to this work.

\section{Acknowledgments}

This work was supported by the National Natural Science Foundation of China (81171798, 81121004, and 81230041), 
Beijing Municipal Natural Science Foundation (7142124), and the National Basic Science and Development Program (973 Program, 2012CB518105).

\section{References}

[1] S. B. P. Chargé and M. A. Rudnicki, "Cellular and molecular regulation of muscle regeneration," Physiological Reviews, vol. 84, no. 1, pp. 209-238, 2004.

[2] C. D. Markert, M. A. Merrick, T. E. Kirby, and S. T. Devor, "Nonthermal ultrasound and exercise in skeletal muscle regeneration," Archives of Physical Medicine and Rehabilitation, vol. 86, no. 7, pp. 1304-1310, 2005.

[3] Y. Xu, T. C. Liu, and L. Cheng, "Photobiomodulation process," International Journal of Photoenergy, vol. 2012, Article ID 374861, 7 pages, 2012.

[4] T. Karu, "Laser biostimulation: a photobiological phenomenon," Journal of Photochemistry and Photobiology B: Biology, vol. 3, no. 4, pp. 638-640, 1989.

[5] G. Litscher, "Cerebral and peripheral effects of laserneedle®stimulation," Neurological Research, vol. 25, no. 7, pp. 722-728, 2003.

[6] D. Hawkins and H. Abrahamse, "Phototherapya treatment modality for wound healing and pain relief," African Journal of Biomedical Research, vol. 10, no. 2, pp. 99-109, 2007.

[7] H. Ma, Y. X. Li, H. L. Chen, Y. X. Cui, and T. C. Y. Liu, "Effects of low-intensity laser irradiation on wound healing in diabetic rats," International Journal of Photoenergy, vol. 2012, Article ID 838496, 7 pages, 2012.

[8] D. Hawkins-Evans and H. Abrahamse, "Efficacy of three different laser wavelengths for in vitro wound healing," Photodermatology Photoimmunology and Photomedicine, vol. 24, no. 4, pp. 199-210, 2008.

[9] J. Nakano, H. Kataoka, J. Sakamoto, T. Origuchi, M. Okita, and T. Yoshimura, "Low-level laser irradiation promotes the recovery of atrophied gastrocnemius skeletal muscle in rats," Experimental Physiology, vol. 94, no. 9, pp. 1005-1015, 2009.

[10] F. S. Tedesco, A. Dellavalle, J. Diaz-Manera, G. Messina, and G. Cossu, "Repairing skeletal muscle: Regenerative potential of skeletal muscle stem cells," Journal of Clinical Investigation, vol. 120, no. 1, pp. 11-19, 2010.

[11] B. Péault, M. Rudnicki, Y. Torrente et al., "Stem and progenitor cells in skeletal muscle development, maintenance, and therapy," Molecular Therapy, vol. 15, no. 5, pp. 867-877, 2007.

[12] M. A. Rudnicki, P. N. Schnegelsberg, R. H. Stead, T. Braun, H. $\mathrm{H}$. Arnold, and R. Jaenisch, "MyoD or Myf-5 is required for the formation of skeletal muscle," Cell, vol. 75, no. 7, pp. 1351-1359, 1993.

[13] A. Irintchev, M. Langer, M. Zweyer, R. Theisen, and A. Wernig, "Functional improvement of damaged adult mouse muscle by implantation of primary myoblasts," Journal of Physiology, vol. 500, no. 3, pp. 775-785, 1997.

[14] C. Zhang, P. Chen, Y. Fei et al., "Wnt/ $\beta$-catenin signaling is critical for dedifferentiation of aged epidermal cells in vivo and in vitro," Aging Cell, vol. 11, no. 1, pp. 14-23, 2012.

[15] D. Yang, X. Wu, W. Hou, X. Zheng, J. Zheng, and Y. Jiang, "Assessing the therapeutic effect of $630 \mathrm{~nm}$ light-emitting diodes irradiation on the recovery of exercise-induced hand muscle fatigue with surface electromyogram," International Journal of Photoenergy, vol. 2012, Article ID 652040, 8 pages, 2012.
[16] M. Monici, F. Cialdai, F. Ranaldi et al., "Effect of IR laser on myoblasts: a proteomic study," Molecular BioSystems, vol. 9, no. 6, pp. 1147-1161, 2013.

[17] T. C. Y. Liu, D. F. Wu, L. Zhu, P. Peng, L. Liu, and X. B. Yang, "Microenvironment dependent photobiomodulation on function-specific signal transduction pathways," International Journal of Photoenergy, vol. 2014, Article ID 904304, 8 pages, 2014.

[18] T. Karu, "Photobiology of low-power laser effects," Health Physics, vol. 56, no. 5, pp. 691-704, 1989.

[19] T. Karu, "Primary and secondary mechanisms of action of visible to near-IR radiation on cells," Journal of Photochemistry and Photobiology B: Biology, vol. 49, no. 1, pp. 1-17, 1999.

[20] W. A. Kues, M. Anger, J. W. Carnwath, D. Paul, J. Motlik, and H. Niemann, "Cell cycle synchronization of porcine fetal fibroblasts: effects of serum deprivation and reversible cell cycle inhibitors," Biology of Reproduction, vol. 62, no. 2, pp. 412-419, 2000.

[21] P. S. Rabinovitch, M. Kubbies, Y. C. Chen, D. Schindler, and H. Hoehn, "BrdU-Hoechst flow cytometry: a unique tool for quantitative cell cycle analysis," Experimental Cell Research, vol. 174, no. 2, pp. 309-318, 1988.

[22] M. Kitzmann and A. Fernandez, "Crosstalk between cell cycle regulators and the myogenic factor MyoD in skeletal myoblasts," Cellular and Molecular Life Sciences, vol. 58, no. 4, pp. 571-579, 2001.

[23] L. A. Sabourin and M. A. Rudnicki, "The molecular regulation of myogenesis," Clinical Genetics, vol. 57, no. 1, pp. 16-25, 2000.

[24] T. C. Liu, Y. Liu, E. Wei, and F. Li, "Photobiomodulation on stress," International Journal of Photoenergy, vol. 2012, Article ID 628649, 11 pages, 2012. 

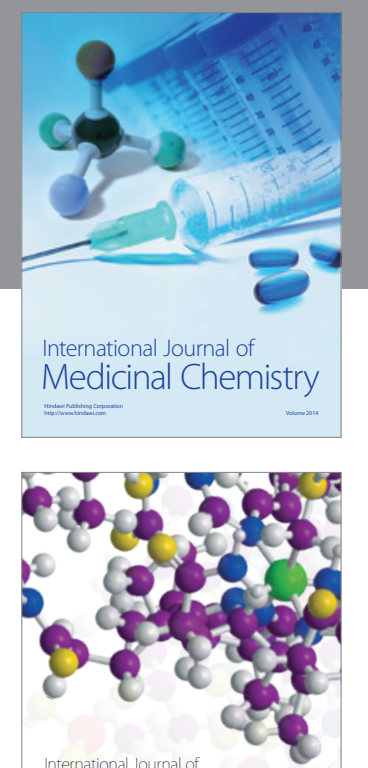

\section{Carbohydrate} Chemistry

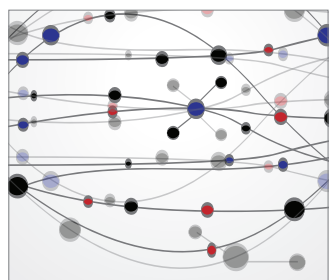

The Scientific World Journal
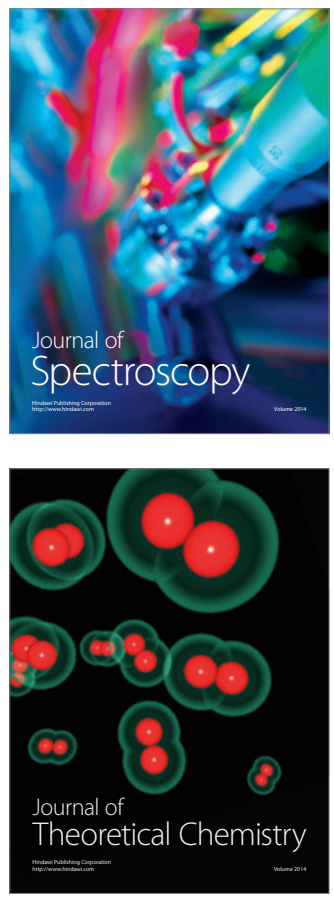
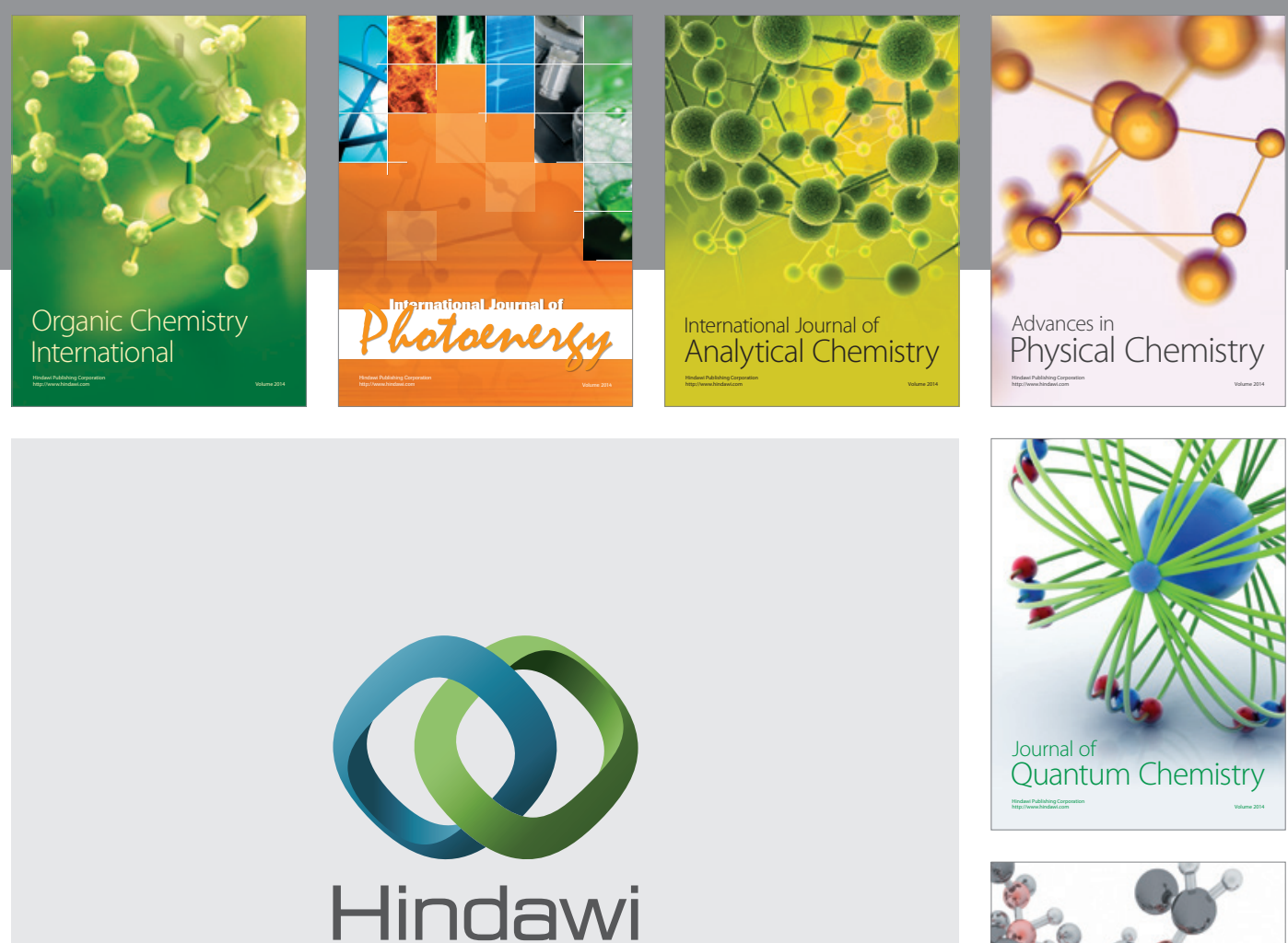

Submit your manuscripts at

http://www.hindawi.com

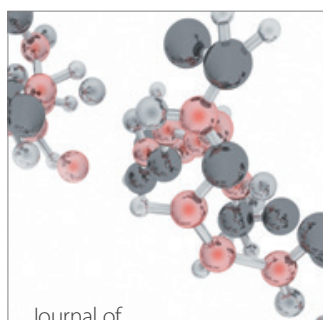

Analytical Methods

in Chemistry

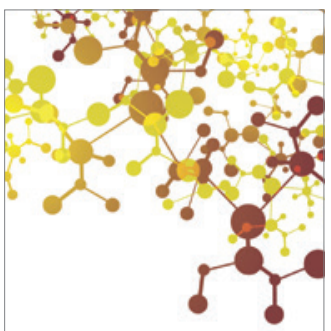

Journal of

Applied Chemistry

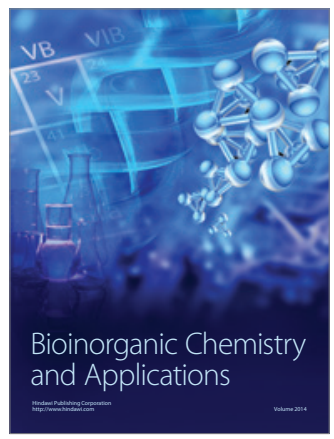

Inorganic Chemistry
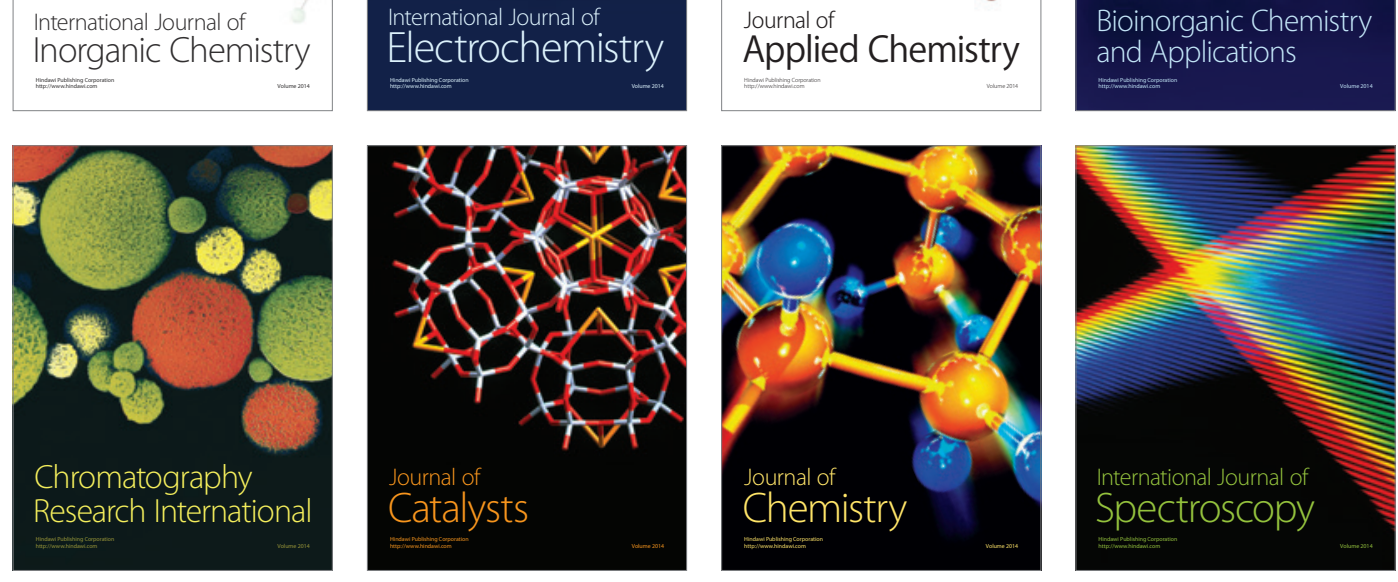